\title{
Endovascular therapy for selected (most non-surgical) intracranial aneurysms in a Brazilian University Hospital
}

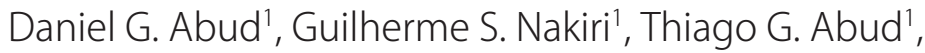 \\ Carlos G. Carlotti Jr. ${ }^{2}$, Benedicto O. Colli ${ }^{2}$, Antonio C. Santos ${ }^{1}$
}

\begin{abstract}
Objective: The objective of this study was to evaluate technical, clinical and angiographic results of a nonsurgical series of intracranial aneurysms treated by endovascular approach at Hospital das Clínicas of Medical School of Ribeirão Preto - University of São Paulo. Method: Between August 2005 and November 2008, 137 aneurysms in 106 patients were endovascularly treated. Of these, 101 were unruptured in 75 patients and 36 aneurysms in 31 patients were treated during the acute phase. The data were prospectively studied. Results: Sixty three aneurysms (46\%) were treated with coils alone, 52 (38\%) with balloon remodeling, 15 (10.9\%) with stent remodeling, and 7 (5.1\%) with therapeutic occlusion of the internal carotid artery. Six clinical complications (5.7\%) were related to the procedures, 3 (2.8\%) transitory and 3 (2.8\%) permanent. Angiographic follow-up was available for 97 aneurysms (70.8\%), clinical monitoring for 77 patients (72.6\%) and telephone contact for 97 (91.5\%). Conclusion: The technical, clinical and angiographic results found in this study are similar to those reported in the literature.

Key words: intracranial aneurysm, endovascular treatment, remodeling technique, socioeconomics, complications.
\end{abstract}

Tratamento endovascular de aneurismas selecionados (maioria não cirúrgicos) em um hospital universitário brasileiro

\section{RESUMO}

Objetivo: Nosso objetivo foi avaliar os resultados técnicos, clínicos e angiográficos de uma série de aneurismas intracranianos não cirúrgicos tratados por via endovascular no Hospital das Clínicas da Faculdade de Medicina de Ribeirão Preto da Universidade de São Paulo e comparar com os dados disponíveis na literatura atualmente. Método: Entre agosto de 2005 e novembro de 2008, 137 aneurismas foram tratados por via endovascular em 106 pacientes. Destes, 101 eram não rotos em 75 pacientes e 36 aneurismas foram tratados em 31 pacientes durante a fase aguda de ruptura. Os dados foram incluídos de maneira prospectiva. Resultados: Sessenta e três aneurismas (46\%) foram tratados com técnica simples, 52 (38\%) com remodelagem por balão, 15 (10,9\%) com remodelagem por stent e $7(5,1 \%)$ por oclusão terapêutica da carótida interna. Seis complicações clínicas ocorreram

Correspondence

Daniel Giansante Abud

Department of Internal Medicine

Division of Radiology

Medical School of Ribeirão Preto

University of São Paulo

Avenida Bandeirantes 3900

14048-090 Ribeirão Preto SP - Brasil

E-mail: dgabud@fmrp.usp.br

Received 27 September 2009

Received in final form 4 March 2010

Accepted 15 March 2010
$(5,7 \%), 3(2,8 \%)$ transitórias e $3(2,8 \%)$ permanentes. Seguimento angiográfico foi realizado para 97 aneurismas $(70,8 \%)$, clínico para 77 pacientes $(70,8 \%)$ e contato telefônico para 97 pacientes $(91,5 \%)$. Conclusão: Os resultados encontrados nesta série, em termos técnicos, clínicos e angiográficos, são semelhantes aos encontrados na literatura.

Palavras-chave: aneurisma intracraniano, tratamento endovascular, técnica de remodelagem, fatores socioeconômicos, complicações.

'Department of Internal Medicine, Division of Radiology, Medical School of Ribeirão Preto, University of São Paulo, Ribeirão Preto SP, Brazil; ${ }^{2}$ Department of Surgery, Division of Neurosurgery, Medical School of Ribeirão Preto, University of São Paulo, Ribeirão Preto SP, Brazil. 
Endovascular treatment of cerebral aneurysms underwent a revolution after the introduction of controlled detachable coils ${ }^{1}$. The first series available in the literature reported the results only for aneurysms not suited for surgery $^{2,3}$. In 2002 the ISAT study ${ }^{4}$ demonstrated at level 1 of evidence that ruptured cerebral aneurysms significantly benefited from endovascular treatment, changing the treatment of cerebral aneurysms all over the world. Unfortunately, this achievement may not be reproducible in many developing countries, since there are no many publications showing their results ${ }^{5}$ and many factors hinder the development of the technique.

Initially restricted to small neck aneurysms, balloon ${ }^{6}$ and stent ${ }^{7,8}$ remodeling, allied to therapeutic occlusion of the internal carotid artery (ICA) ${ }^{9}$ have allowed the treatment of the majority of intracranial aneurysms.

Our study comprises a population of most non-surgical aneurysms treated by endovascular approach at the Medical School of Ribeirão Preto, University of São Paulo, after the recent establishment of interventional neuroradiology discipline. The patients included in the study were considered unsuitable for surgical approach by the vascular neurosurgeons of the institution. Factors related to complications and treatment stability have been analyzed and compared to the results in the literature.

\section{METHOD}

This prospective study period began with the first patient treated in August 2005 and ended with the last treatment included in November 2008. Collected data included demographic information, aneurysms characteristics, treatment strategy, angiographic results, clinical condition at admission and discharge, clinical follow-up and telephone interview. Retreatments and their results were also included.

This research was approved by the ethical committee of our institution and all the patients had their inclusion in the study authorized by an informed consent.

The patients were divided into two groups to analyze the clinical results: with unruptured and ruptured aneurysms. Technical results and stability were analyzed considering the entire group.

Inclusion criteria were: risky location or inaccessible for surgery; patients with critical clinical conditions; multiple aneurysms, one of which already treated surgically, patients with residual aneurysm after surgery, and patient's option for endovascular treatment.

Aneurysms considered suitable for surgery were excluded by the vascular neurosurgical team, without the participation of the interventional neuroradiology in this decision.

All procedures were performed on a monoplane angiographic system without 3-dimensional reconstruction.
The procedures were performed under general anesthesia (GA) and a 5000UI bolus of heparin was administered followed by 2500-3000UI continuously after the digital subtracted angiography (DSA). In the absence of complications, heparin was ceased at the end of the procedure.

Antiplatelets were not used as routine preparation for the endovascular treatment of ruptured or unruptured aneurysms. However, aneurysms treated with stent remodeling received $300 \mathrm{mg}$ aspirin and $75 \mathrm{mg}$ clopidogrel per day 5 days before the procedure, continued for 6 months, followed by aspirin alone for 1 year.

After analyzing the aneurysm, one of these 4 therapeutic alternatives was chosen: [1] coils alone for small neck aneurysms, [2] balloon remodeling for wide neck aneurysms, [3] stent remodeling for large and giant wide neck aneurysms or, [4] therapeutic occlusion for aneurysms unsuited for selective treatment.

The devices used for endovascular procedures in our hospital are purchased through public licitation. The coils used were GDC (Boston Scientific, Fremont, Calif) and Saphire (ev3, Irvine, Calif). Remodeling balloons were Sentry (Boston Scientific), Hyperglide and Hyperform (ev3). Stent remodeling was performed with Neuroform3 (Boston Scientific).

DSA was performed 6, 18 and 36 months after the treatment. At the time of each angiographic control, a clinical assessment was conducted.

Angiographic occlusion rate were graduated as: complete (class-I), neck-remnant (class-II) or incomplete (class-III) according to the Raymond's classification ${ }^{10}$. They were clinically evaluated according to the modified Rankin's scale (mRS). After inclusion of all patients in the study, we have tried to contact each one of them by telephone to assess their clinical condition.

At the end of the 36-month period, in the absence of recurrence, the patient was discharged. For major recurrences, a retreatment was performed. In our casuistic, all aneurysms with major recurrences were retreated.

Factors possibly related to complications were analyzed such as: patients age, aneurysm size and neck size, and type of treatment, as well as factors possibly related to recurrence such as: unsatisfactory angiographic result, aneurysm size and neck, and type of treatment. Statistical analyses were performed using Fisher's exact test, with a $5 \%$ significance level.

\section{RESULTS}

\section{Patients and aneurysms}

From August 2005 to November 2008, 137 saccular aneurysms were treated in 106 patients, 101 unruptured in 75 patients and 36 aneurysms in 31 patients were treated during the acute phase of rupture. Patients included 
Table 1. Patients.

\begin{tabular}{lc}
\hline $\begin{array}{l}\text { Age group } \\
<29 \text { years }\end{array}$ & $3(2.8 \%)$ \\
30 to 39 years & $10(9.4 \%)$ \\
40 to 49 years & $23(21.7 \%)$ \\
50 to 59 years & $36(34 \%)$ \\
60 to 69 years & $23(21.7 \%)$ \\
$\geq 70$ years & $11(10.4 \%)$ \\
Gender & \\
Female & $84(79.2 \%)$ \\
Male & $22(20.8 \%)$ \\
Ruptured / Unruptured & \\
Unruptured & $75(70.8 \%)$ \\
Ruptured & $31(29.2 \%)$ \\
\hline
\end{tabular}

Table 2. Aneurysms.

\begin{tabular}{|c|c|}
\hline \multicolumn{2}{|l|}{ Size } \\
\hline$<7 \mathrm{~mm}$ & $42.3 \%(58)$ \\
\hline $7-12 \mathrm{~mm}$ & $34.3 \%(47)$ \\
\hline $13-24 \mathrm{~mm}$ & $10.2 \%(14)$ \\
\hline$\geq 25 \mathrm{~mm}$ & $13.1 \%(18)$ \\
\hline \multicolumn{2}{|l|}{ Neck } \\
\hline$\leq 4 \mathrm{~mm}$ & $39.4 \%(54)$ \\
\hline$>4 \mathrm{~mm}$ & $60.6 \%(83)$ \\
\hline \multicolumn{2}{|l|}{ Location } \\
\hline \multicolumn{2}{|l|}{ Anterior circulation } \\
\hline Acom & $7.3 \%(10)$ \\
\hline Pericallosal artery & $1.5 \%(2)$ \\
\hline MCA & $9.5 \%(13)$ \\
\hline Anterior choroidal artery & $1.5 \%(2)$ \\
\hline ICA bifurcation & $2.9 \%(4)$ \\
\hline Ophthalmic artery & $15.3 \%(21)$ \\
\hline Pcom & $18.2 \%(25)$ \\
\hline Superior hypophyseal artery & $10.2 \%(14)$ \\
\hline Cavernous segment & $13.1 \%(18)$ \\
\hline Persistent trigeminal artery & $0.7 \%(1)$ \\
\hline \multicolumn{2}{|l|}{ Posterior circulation } \\
\hline Basilar tip & $10.9 \%(15)$ \\
\hline Basilar trunk & $3.6 \%(5)$ \\
\hline PICA & $2.2 \%(3)$ \\
\hline Posterior cerebral artery & $1.5 \%(2)$ \\
\hline Superior cerebellar artery & $1.5 \%(2)$ \\
\hline \multicolumn{2}{|l|}{ Treatment strategy } \\
\hline Simple technique & $46 \%(63)$ \\
\hline Balloon remodeling & $38 \%(52)$ \\
\hline Stent remodeling & $10.9 \%(15)$ \\
\hline Therapeutic occlusion & $5.1 \%(7)$ \\
\hline
\end{tabular}

Acom: anterior co mmunicating artery; MCA: middle cerebral artery; ICA: internal carotid artery; Pcom: posterior co mmunicating artery; PICA: posterior inferior cerebellar artery.
84 female and 22 male, their ages ranging from 22 to 80 years (mean age, 54 years) (Table 1 ).

A hundred and ten aneurysms were located in the anterior $(80.3 \%)$ and $27(19.7 \%)$ in the posterior circulation; $18(13.1 \%)$ in the cavernous segment, 35 (28.5\%) were paraclinoid and only 13 (9.5\%) were located in the middle cerebral artery (MCA) and 10 (7.3\%) in the anterior communicating artery (Acom).

We found 58 (42.3\%) small aneurysms ( $<7 \mathrm{~mm}), 47$ (34.3\%) medium (7-12 mm), 14 (10.2\%) large (13-24 mm) and 18 (13.1\%) giant ( $\geq 25 \mathrm{~mm}$ ). The mean-size was 10.8 $\mathrm{mm}$. Out of the 101 unruptured aneurysms, 41 (40.6\%) were small, 29 (28.7\%) medium, 13 (12.9\%) large, and 18 (17.8\%) giant. Among the 36 ruptured aneurysms, 17 (47.2\%) small, 18 (50\%) medium, and 1 (2.8\%) large. Data regarding aneurysms characteristics and locations are demonstrated in Table 2.

\section{Treatment strategy}

Sixty three aneurysms (46\%) were treated with coils alone, 52 (38\%) with balloon remodeling, 15 (10.9\%) with stent remodeling and 7 (5.1\%) with therapeutic occlusion (Table 2). Out of the 101 unruptured aneurysms, 33 (32.7\%) were treated with coils alone, 46 (45.5\%) with balloon remodeling, 15 (14.9\%) with stent remodeling and 7 (6.9\%) with therapeutic occlusion. Among the 36 ruptured aneurysms, 30 (84.2\%) were treated with coils alone, and $6(15.8 \%)$ with balloon remodeling.

\section{Complications}

There were 9 (6.6\%) technical complications, 6 (4.4\%) asymptomatic and $3(2.2 \%)$ symptomatic. Complications were thromboembolic for 5 aneurysms, stretched coils for 2 , rupture for 1 and infected hematoma at the puncture site for 1.

No technical complications occurred for aneurysms treated with stent remodeling or therapeutic occlusion. Four (7.7\%) technical complications occurred in the group of aneurysms treated with balloon remodeling and $5(7.9 \%)$ in the group treated with coils alone.

Aneurysm ( $\mathrm{p}=0.7138)$ or neck size $(\mathrm{p}=0.3165)$, as well as treatment strategy $(\mathrm{p}=0.8674)$ did not influence the complication rates as shown in Table 3.

Clinical complications occurred in $6(5.7 \%)$ patients among the 106, 3 (2.8\%) transitory and 3 (2.8\%) permanent. Two permanent clinical complications were due to ischemia related to thromboembolic event and a third one due to a stretched coil in a patient with ruptured aneurysm who was kept under antiplatelets, and had a hemorrhagic complication during a ventricular shunt placement.

There were no difference in terms of clinical complication rates among the age groups $(\mathrm{p}=0.8874)$ (Table 4). 
Table 3. Technical complications.

\begin{tabular}{lc}
\hline & Complications \% (n) \\
\hline Technical complications & $6.6 \%(9)$ \\
Symptomatic & $2.2 \%(3)$ \\
Asymptomatic & $4.4 \%(6)$ \\
Aneurysm size & \\
$<7 \mathrm{~mm}$ & $8.6 \%(5)$ \\
$7-12 \mathrm{~mm}$ & $6.4 \%(3)$ \\
$13-24 \mathrm{~mm}$ & $7.1 \%(1)$ \\
$\geq 25 \mathrm{~mm}$ & $0 \%(0)$ \\
& $\mathrm{p}=0.7138$ \\
Neck size & \\
$\leq 4 \mathrm{~mm}$ & $9.3 \%(5)$ \\
$>4$ mm & $4.8 \%(4)$ \\
& $\mathrm{p}=0.3165$ \\
Treatment strategy & \\
Coils alone & $7.9 \%(5)$ \\
Balloon remodeling & $7.7 \%(4)$ \\
Stent remodeling & $0 \%(0)$ \\
Therapeutic occlusion & $0 \%(0)$ \\
& $\mathrm{p}=0.8674$ \\
\hline
\end{tabular}

\section{Clinical results}

Out of the 75 patients harboring 101 unruptured aneurysms, 72 (96\%) were independent at the admission (mRS 0-2). Upon hospital discharge, there was clinical deterioration in 1 patient (1.3\%).

Clinically, 61 patients (81.3\%) were evaluated once, 22 (29.3\%) twice and 4 (5.3\%) three times (mean follow-up, 12.1 months). Comparing to admission, there was clinical deterioration in 1 patient who became dependent, and improvement of 1 patient who became independent.

Telephone interviews were possible for 74 (98.7\%) of the patients harboring unruptured aneurysms (mean follow-up, 22 months), where 70 (94.6\%) were independent. Comparing to admission, one patient became independent, and one patient continued to be dependent. Another patient became dependent after meningioma surgery and one died of heart attack.

Subarachnoid hemorrhage for ruptured aneurysms, was graded as Fisher 1 for 1 (3.2\%) patient, 2 for 4 (12.9\%) patients, 3 for 7 (22.6\%) and 4 for 19 (61.3\%). According to Hunt and Hess's scale (HH), 19 (61.3\%) were in good grade (HH 1-3) and 12 (38.7\%) bad grade (HH 4-5).

At discharge, 16 patients (51.6\%) were independent, 6 (19.4\%) dependent and 9 (29\%) died.

Among the 19 patients in good clinical conditions, $15(78.9 \%)$ were independent at the hospital discharge, 2 (10.5\%), were dependent and 2 (10.5\%) died, whereas among the 12 patients in poor conditions, 1 (8.3\%) was independent at the time of discharge, 4 (33.3\%) were
Table 4. Clinical complications.

\begin{tabular}{lc}
\hline & Complications \% (n) \\
\hline Clinical complications & $5.7 \%(6)$ \\
Permanent & $2.8 \%(3)$ \\
Transitory & $2.8 \%(3)$ \\
Age & \\
$\quad<40$ & $0 \%(0)$ \\
$40-49$ & $4.3 \%(1)$ \\
$50-59$ & $8.3 \%(3)$ \\
$60-69$ & $4.3 \%(1)$ \\
$\geq 70$ & $9.1 \%(1)$ \\
& $\mathrm{p}=0.8874$ \\
\hline
\end{tabular}

Table 5. Major recurrences.

\begin{tabular}{lc}
\hline & Major recurrences $\%(\mathrm{n})$ \\
\hline Total & $8.2 \%(8)$ \\
Immediate result & \\
Class I & $2.4 \%(2)$ \\
Class II & $50 \%(4)$ \\
Class III & $40 \%(4)$ \\
& $\mathrm{p}<0.0001$ \\
Aneurysm size & \\
$<7$ mm & $0 \%(0)$ \\
$7-12$ mm & $3.3 \%(1)$ \\
13-24 mm & $16.7 \%(2)$ \\
& $33.3 \%(5)$ \\
Neck size & $\mathrm{p}<0.0001$ \\
$<4$ mm & \\
$\geq 4$ mm & $0 \%(0)$ \\
Treatment strategy & $13.1 \%(8)$ \\
Coils alone & $\mathrm{p}=0.0241$ \\
Balloon remodeling & \\
Thent remodeling & $10.2 \%(4)$ \\
\hline
\end{tabular}

dependent and 7 (58.3\%) died. Among the 31 patients treated in this group, 16 (72.7\% of survivors) were clinically evaluated once, 8 (25.8\%) twice and 1 (3.2\%) three times and 14 were independent (mean follow-up, 14.5 months).

All of the 22 surviving patients after discharge could be contacted by telephone (mean follow-up, 21.5 months). Eighteen (58.06\%) were independent, 2 dependent and 2 died of clinical complications. Among the 19 patients in good conditions, only 1 was dependent, representing $84.2 \%$ of good results. No patient had any rebleeding episode after aneurysm treatment. 


\section{Angiographic results and follow-up}

Angiographic result class I was obtained at the end of treatment for 118 aneurysms (86.13\%), class II for 9 (6.57\%) and class III for 10 (7.30\%).

Angiographic controls were carried out for 97 aneurysms (76.98\% of the surviving patients) (mean follow-up, 12.3 months). Thirty-six aneurysms ( $26.28 \%$ of total) were controlled twice and 7 (5.11\%) three times.

Among the 97 controlled aneurysms, 8 (8.25\%) were retreated. As all major recurrences were retreated, this group was considered for analysis. No complications occurred in the retreatments.

Retreatment had strong correlations with immediate angiographic result $(\mathrm{p}<0.0001)$, aneurysm size $(\mathrm{p}<0.0001)$ and neck size $(\mathrm{p}=0.0241)$, as shown in Table 5 .

No retreatment was required for the 13 aneurysms treated by stent remodeling or for the 5 aneurysms treated by therapeutic occlusion. Among the 39 aneurysms treated by coils alone, 4 (10.3\%) required retreatment; among the 40 treated by balloon remodeling, 4 (10\%) required retreatment, without statistical relevance $(\mathrm{p}=0.8518)$. However, when only large and giant aneurysms were analyzed, we verified that 4 (66.7\%) of the 6 aneurysms treated by coils alone and $3(42.9 \%)$ of the 7 treated by balloon remodeling required retreatment. None of the 9 aneurysms treated with stent remodeling, or of the 5 aneurysms with therapeutic occlusion, required retreatment. The treatment was more stable for stent remodeling and therapeutic occlusion in this group ( $\mathrm{p}=0.0061)$.

\section{DISCUSSION}

Representing a series of most non-surgical, a large number of our patients were older than 60 years-old (32.1\%). Frequent locations such as MCA and Acom were rarely found, and difficult surgical locations were more common, such as posterior circulation, paraclinoid and cavernous aneurysms. Among the patients treated during the acute phase, $38.7 \%$ presented poor clinical condition.

There were a large number of aneurysms treated with balloon (38\%) and stent remodeling (10.9\%). In the group of unruptured aneurysms, only $32.7 \%$ were treated with coils alone, $45.5 \%$ with balloon remodeling, $14.9 \%$ with stent and $6.9 \%$ with therapeutic occlusion.

The first series available in the literature report on the results of aneurysm treatment using only the simple technique, restricted to small neck aneurysms. After the introduction of balloon remodeling by Moret ${ }^{6}$, the embolization of wide-necked aneurysms became possible in safe conditions. Stent remodeling was initially performed with balloon expandable stents, with high complication rates ${ }^{7}$. Self-expandable stents adapted for intracranial circulation have recently shown better results ${ }^{8}$ and became an important device in the treatment of intracranial aneurysms.
The therapeutic occlusion of the ICA was described in $1974^{9}$ to deal with complex aneurysms. The occlusion test protocol varies markedly in the literature. We used the angiographic test occlusion described by Abud et al. ${ }^{11}$, without clinical evaluation.

The evaluation of the endovascular treatment of unruptured aneurysms, in the recent ATENA study ${ }^{12}$, presented a proportion of endovascular techniques similar to ours, in which $98.4 \%$ of the aneurysms were selectively treated, $54.5 \%$ with coils alone, $37.3 \%$ with balloon remodeling and $7.3 \%$ with stent remodeling.

According to our results, thromboembolic events were the most frequent complication, while the hemorrhagic ones were rare.

Thromboembolic complications incidence ranges between $2.5 \%$ and $11 \%$ in the literature, with clinical consequences in $2.5 \%$ to $5.5 \%$ of the patients ${ }^{13,14}$.

Embolic events related to embolization were initially treated with thrombolytic agents, with disastrous consequences to patients with ruptured aneurysms ${ }^{4}$. Antiplatelets, abxicimab ${ }^{15}$ and tirofiban ${ }^{16}$, have been recently introduced dealing with these complications with good results.

Aneurysm perforation is the most feared complication; however, it does not always have serious consequences.

In a meta-analysis of 17 retrospective studies ${ }^{17}$, it was found a higher risk of perforation for ruptured aneurysms (4.1\%) compared to unruptured ones (0,5\%), leading to a morbi-mortality of $38 \%$ and $29 \%$ respectively.

We have found no statistically significant differences in terms of complications for the different techniques. No permanent complications occurred in the groups of aneurysms treated with stent remodeling or therapeutic occlusion, but the small number of patients included in these groups should be considered. The rates of technical complications were similar for aneurysms treated with coils alone and balloon remodeling.

In Spelle's ${ }^{18}$ recent review of the available publications on the endovascular treatment of unruptured aneurysms, the morbidity rate associated with procedures ranged between 3 and $6 \%$, with $1.5 \%$ mortality. In the ATENA study ${ }^{12}$ the morbidity-mortality rates were 1.7 and $1.4 \%$ respectively. In our study we found $1.3 \%$ permanent morbidity and $0 \%$ mortality related to the procedures for unruptured aneurysms.

In the ISAT study ${ }^{4}, 88 \%$ of the included patients were in good conditions and $75 \%$ were independent after treatment. These findings are similar to our population of ruptured-aneurysm patients in good conditions, in which $78.9 \%$ were independent at the discharge and $84.2 \%$ in the follow-up.

The incidence of angiographic recurrence varies in 
the literature, between $14.7 \%$ and $33.6 \%{ }^{19,20}$. Retreatment rate is more stable, around $10 \%{ }^{20,21}$, and is related to larger recurrences.

Piotin ${ }^{19}$ published a study about aneurysm recurrence, evaluating 255 selectively embolized aneurysms with at least one angiographic control. Seventy-three aneurysms (28.6\%) presented recurrence and 14 (5.5\%) were retreated. The length of follow-up was the most important factor

Raymond ${ }^{20}$ studied 501 ruptured aneurysms, and found recurrence in 33.6\% of the cases. Major recurrences were found in $20.7 \%$ and half of them were retreated (10.2\%). The factors implied with recurrence were aneurysms treated in the acute stage, aneurysm size, neck size, incomplete immediate result and follow-up duration.

In the angiographic follow-up of the ISAT study ${ }^{22}$, retreatment was necessary for $10.6 \%$ of the patients that were submitted to control angiograms.

If only large and giant aneurysms are considered, which represent a challenge for the endovascular technique due to their high rate of recanalization ${ }^{23}$, we have found that treatment with stent remodeling and therapeutic occlusion of the ICA were statistically more stable than simple technique and balloon remodeling. However, these groups were smaller than the groups of aneurysms treated with balloon remodeling or by simple techniques. The development of new devices such as the flow-diverter stents may improve the stability over time in these groups ${ }^{24}$.

Our retreatment rate was $8.2 \%$ considering the 97 aneurysms that were submitted to at least one angiographic control and is similar to other results in the literature.

Although recurrence rate is always brought to attention in the discussion of endovascular embolization, it is well established in the literature that complications related to retreatment are rare.

A recent study ${ }^{25}$ reported 100 retreated aneurysms in 99 patients and found thromboembolic events in 3 procedures, without clinical implications.

The contribution of endovascular therapy in dealing with cerebral aneurysms is undeniable. Our series of patients with high surgical risk showed good results and reveals that, in our context, embolization can be safely applied.

In conclusion, endovascular treatment is a viable option within the Brazilian public health care system and must be taken into account when treatment options are discussed for such patients. The results for our series of predominating non-surgical aneurysms are similar to those available in current publications.

Aneurysm size, neck size, patient age and treatment strategy did not alter the complication rates.

Sac and neck dimensions as well as immediate angiographic results are related to recurrence.

\section{REFERENCES}

1. Guglielmi G, Vinuela F, Sepetka I, Macellari V. Electrothrombosis of saccular aneurysms via endovascular approach. Part 1: Electrochemical basis, technique, and experimental results. J Neurosurg 1991;75:1-7.

2. Klein GE, Szolar DH, Leber KA, Karaic R, Hausegger KA. Basilar tip aneurysm: endovascular treatment with guglielmi detachable coils: midterm results. Radiology 1997;205:191-196.

3. van der Schaaf IC, Brilstra EH, Buskens E, Rinkel GJ. Endovascular treatment of aneurysms in the cavernous sinus: a systematic review on balloon occlusion of the parent vessel and embolization with coils. Stroke 2002:33:313-318.

4. Molyneux A, Kerr R, Stratton I, et al. International subarachnoid aneurysm trial (ISAT) of neurosurgical clipping versus endovascular coiling in 2143 patients with ruptured intracranial aneurysms: a randomised trial. Lancet 2002;360:1267-1274.

5. Souza JR, Otoch M, Ribeiro SP, et al. Endovascular treatment of cerebral aneurysms: a retrospective study of 163 embolized aneurysms. Arq Neuropsiquiatr 2007;65:411-415.

6. Moret J, Cognard C, Weill A, Castaings L, Rey A. [reconstruction technic in the treatment of wide-neck intracranial aneurysms. Long-term angiographic and clinical results. A propos of 56 cases]. J Neuroradiol 1997;24:30-44.

7. Kessler IM, Mounayer C, Piotin M, Spelle L, Vanzin JR, Moret J. The use of balloon-expandable stents in the management of intracranial arterial diseases: a 5-year single-center experience. AJNR Am J Neuroradiol 2005;26: 2342-2348.

8. Fiorella D, Albuquerque FC, Han P, McDougall CG. Preliminary experience using the neuroform stent for the treatment of cerebral aneurysms. Neurosurgery 2004:54:6-17.

9. Serbinenko FA. Balloon catheterization and occlusion of major cerebral vessels. J Neurosurg 1974;41:125-145.

10. Roy D, Milot G, Raymond J. Endovascular treatment of unruptured aneurysms. Stroke 2001;32:1998-2004.

11. Abud DG, Spelle L, Piotin M, Mounayer C, Vanzin JR, Moret J. Venous phase timing during balloon test occlusion as a criterion for permanent internal carotid artery sacrifice. AJNR Am J Neuroradiol 2005;26:2602-2609.

12. Pierot L, Spelle L, Vitry F. Immediate clinical outcome of patients harboring unruptured intracranial aneurysms treated by endovascular approach: results of the ATENA study. Stroke 2008;39:2497-2504.

13. Cognard C, Weill A, Castaings L, Rey A, Moret J. Intracranial berry aneurysms: angiographic and clinical results after endovascular treatment. Radiology 1998;206:499-510.

14. Vinuela F, Duckwiler G, Mawad M. Guglielmi detachable coil embolization of acute intracranial aneurysm: perioperative anatomical and clinical outcome in 403 patients. J Neurosurg 1997;86:475-482.

15. Mounayer C, Piotin M, Baldi S, Spelle L, Moret J. Intraarterial administration of abciximab for thromboembolic events occurring during aneurysm coil placement. AJNR Am J Neuroradiol 2003;24:2039-2043.

16. Bruening R, Mueller-Schunk S, Morhard D, et al. Intraprocedural thrombus formation during coil placement in ruptured intracranial aneurysms: treatment with systemic application of the glycoprotein iib/iiia antagonist tirofiban. AJNR Am J Neuroradiol 2006;27:1326-1331.

17. Cloft HJ, Kallmes DF. Cerebral aneurysm perforations complicating therapy with guglielmi detachable coils: a meta-analysis. AJNR Am J Neuroradiol 2002;23:1706-1709.

18. Spelle L, Pierot L. [Endovascular treatment of non-ruptured intracranial aneurysms: critical analysis of the literature]. J Neuroradiol 2008;35:116-120.

19. Piotin M, Spelle L, Mounayer C, et al. Intracranial aneurysms: treatment with bare platinum coils: aneurysm packing, complex coils, and angiographic recurrence. Radiology 2007;243:500-508.

20. Raymond J, Guilbert F, Weill A, et al. Long-term angiographic recurrences after selective endovascular treatment of aneurysms with detachable coils. Stroke 2003:34:1398-1403.

21. Sluzewski M, van Rooij WJ, Rinkel GJ, Wijnalda D. Endovascular treatment of ruptured intracranial aneurysms with detachable coils: Iong-term clinical and serial angiographic results. Radiology 2003;227:720-724.

22. Campi A, Ramzi N, Molyneux AJ, et al. Retreatment of ruptured cerebral aneurysms in patients randomized by coiling or clipping in the international subarachnoid aneurysm trial (ISAT). Stroke 2007:38:1538-1544.

23. Parkinson RJ, Eddleman CS, Batjer HH, Bendok BR. Giant intracranial aneurysms: endovascular challenges. Neurosurgery 2006:59:103-113.

24. Lylyk P, Miranda C, Ceratto R, et al. Curative endovascular reconstruction of cerebral aneurysms with the pipeline embolization device: the Buenos Aires experience. Neurosurgery 2009;64:632-642.

25. Renowden SA, Koumellis P, Benes V, Mukonoweshuro W, Molyneux AJ, McConachie NS. Retreatment of previously embolized cerebral aneurysms: the risk of further coil embolization does not negate the advantage of the initial embolization. AJNR Am J Neuroradiol 2008:29:1401-1404. 